\title{
Etiologies of bloodstream infection and antimicrobial resistance: A cross sectional study among patients in a tertiary hospital, Northern Tanzania
}

\author{
Ibrahim Ireneus Mauki ${ }^{a}$ Jesca Deogratias Williama, Henry Lucas Mlaya, Adonira Tajaeli Saroa, b, c, \\ Samwel Ole Saringed, Sixbert Isdory Mkumbaye ${ }^{a}, b$, Debora Charles Kajeguka ${ }^{a}$ \\ Kilimanjaro Christian Medical University College, Moshi, Tanzania \\ 'Kilimanjaro Christian Medical Centre, Moshi, Tanzania \\ 'Central South University, Xiangya School of Basic Medical Sciences, Hunan Province, Changsha City, 410078, China \\ Kampala International University in Tanzania \\ *Correspondence to Dr. Debora Charles Kajeguka (dkajeguka@gmail.com)
}

\begin{abstract}
Background: Bloodstream infections are important causes of morbidity and mortality in people of all age groups, especially in sub-Saharan Africa. In Tanzania, a recent report indicates that case fatality rate of $37 \%$ is attributed to bloodstream infections. The aim of this study was to determine the prevalence and factors associated with bloodstream infections as well as to determine resistance pattern of bacterial isolates among patients visiting Kilimanjaro Christian Medical Centre (KCMC).

Methods: A cross-sectional study was conducted from April to June 2019 at KCMC. A total of 200 patients were included in the study. Blood samples were collected for culture, malaria rapid test, typhoid and brucella tests. Clinical features, comorbid conditions and patients' hospitalization data were recorded in the questionnaire. Logistic regression was used to examine the factors associated with bloodstream infections. Predictors of the outcome were considered significant at $p<0.05$.

Results: The prevalence of bloodstream infections was 52(26\%). Participants with stomachache had less odds of having bloodstream infections as compared to other patients with symptoms ( $A O R=0.22,5.33,95 \% \mathrm{Cl}=0.05-0.97 ; \mathrm{p}=0.04)$. Of the 41 identified isolates, Staphylococcus aureus showed the highest rates of resistance for Meropenem $8(88.8 \%)$, Cefotaxime $6(66.6 \%)$, Amikacin 6 (66.6\%), Gentamicin 6 (66.6\% (and Imipenem (6 (66.6\%). The lowest level of resistance was observed in Ceftriaxone $1(11.1 \%)$.

Conclusion: Bloodstream infections were highly prevalent in this sample (26\%). Staphylococcus spp was the most commonly isolated organism and exhibited a high resistance rate to most antibiotics. This calls for increased and coordinated efforts to improve the identification, treatment and management of bloodstream infections and antimicrobial resistance, thereby improving clinical practice.
\end{abstract}

\section{INTRODUCTION}

\section{$\mathrm{B}$}

dstream infections (BSIs) are important causes of morbidity and mortality among children and adults in most African Countries ${ }^{1-3}$. In a meta-analysis on community-acquired BSI in Africa, the mean mortality rate was reported to be $18.1 \%^{2}$. In 2007, a study done at Muhimbili National Hospital, Dar es Salaam among a pediatric population, reported mortality rates of $20.2 \%, 43.5 \%$ and $16.7 \%$ for malaria, gram-negative BSIs and gram-positive BSIs respectively ${ }^{4}$. More recently findings from the same hospital indicated a case mortality rate of $37 \%$ among patients of all age groups 5 . The prevalence of BSIs ranges from $13 \%$ to $14.5 \%$ in different parts of the Tanzania $^{4,6,7}$.

There are increased reports of antimicrobial resistance (AMR) among patients with $\mathrm{BSI}^{7-10}$. More importantly, rates of BSI are increasing owing to multidrug-resistant extended-spectrum betalactamase $\quad(\mathrm{ESBL})^{8,11,12}$, methicillinresistant Staphylococcus aureus (MRSA) ${ }^{13}$, and Vancomycin resistance ${ }^{14}$.

In Tanzanian, most of BSIs are misdiagnosed as malaria infections ${ }^{1}$. This is due to the lack of capacity to properly identify the cause of febrile illness ${ }^{15}$. Consequently, Clinicians often rely on clinical features 
to guide the treatment of patient who present with febrile illness ${ }^{16}$ which is less accurate in identifying BSIs, hence risking poor clinical outcomes and promotion of $\mathrm{AMR}^{17}$.

Various factors have been found to be associated with BSI, including; socio-demographics, comorbid conditions, prior hospitalization and recent exposures $^{7}$. Comorbid conditions found to be associated with BSI include: acute and chronic renal failure, hepatic disease, diabetes, hypertension, congestive heart failure, and intravenous drug abuse ${ }^{18}$.

However, there is limited information about BSIs in Northern Tanzania. Specifically, the prevalence, level of resistance and factors associated have not been documented. Notably, the extent of AMR in most BSIs has not been studied. Understanding BSI at KCMC, resistance patterns, and the factors associated with BSIs will guide clinical management and appropriate antibiotic use. Therefore, the present study was designed to document prevalence, factors associated with BSI and resistance patterns of bacteria isolated among patients with BSI.

\section{METHODS}

\section{Study design and area}

This was a hospital-based cross-sectional study. The study was conducted at KCMC referral hospital, located in the foothills of the snowcapped Mount Kilimanjaro in Tanzania (http://www.kcmc.ac.tz/). KCMC has a 650-bed capacity and the second largest consultant referral university teaching hospital in the country serving over 15 million people from northern and central regions of Tanzania, attending more than $800-1000$ outpatients daily.

\section{Study population and inclusion criteria}

The study included all out- and inpatients suspected with BSI. The study included patients of all age suspected of having bacterial or malarial infections. All patients who were critical ill, mentally unfit, and not able to communicate were excluded from the study.

\section{Sample Size Estimation}

Samples was calculated based on the following formula:

$$
\mathrm{N}=\frac{\mathrm{Z}_{\mathrm{p}}^{2}(1-\mathrm{p})}{\varepsilon^{2}}
$$

Where: $\mathrm{N}=$ Sample size, $\mathrm{Z}=$ Level of confidence (1.96), $\mathrm{P}=$ proportion previous prevalence $(13.4 \%) 6$ and $\varepsilon=$ Margin error $(5 \%)$, plus $10 \%$ non-response. Sample size $=1.962 * 0.134 *(1-0.134) /(0.05) 2$. The minimum sample size was $178+10 \%=196$, we rounded up to give a sample size approximation of 200 patients.

\section{Data collection \\ Interview}

Data was collected by three research assistants who were experienced in data collection. All information was collected during face-to-face interviews using questionnaire in Swahili language. The interview lasted between 30 and 45 minutes. The interviews for Out-patients were conducted in a private room where no one other than the research assistant and the patients and/or guardian were allowed to be present. For inpatient, bedside interviews were conducted. The children's information's were given by their parents/ guardians.

The questionnaire had three sections. Section 1: recorded patient's socio-demographic characteristics such as age, sex, occupation, level of education, income per month and residence. Section 2: assessed patient's clinical features and conditions. Other patients' information such as clinical outcomes were retrieved from the patient's medical records. Section 3: collected details on antibiotic usage, and lastly section 4 collated the laboratory investigations

\section{Pre-testing of the questionnaire}

To maximize its validity, the questionnaire was pretested on appropriate respondents before distribution. Interviews were conducted in five (5) patients to examine how patients understood and responded to the questions. In addition to the pilot, two experts in the field of survey design approved the quality of the questionnaire. After the pretest, adjustments in phrasings were made as necessary so that the questionnaire was simple and easily understood.

\section{Blood sample collection and Blood culture}

Venous blood was drawn aseptically from each patient. A total of $2-5 \mathrm{ml}$ of blood was collected in BD BACTEC bottles from pediatrics patients (BD BACTEC Peds PlusTM/F Culture Vials, Becton Dickinson and Company) and $8-10 \mathrm{ml}$ from adults (BD BACTEC Plus Aerobic/F Culture Vials, Becton Dickinson and Company). Blood samples were immediately transported at room temperature to the KCMC clinical laboratory and incubated in the BACTEC machine for further investigation. Blood samples were incubated in BD BACTEC machine for a maximum of 5 days. Positive blood cultures were inoculated on Blood agar, Chocolate agar (both from HI Media Laboratories, Mumbai, India), and MacConkey agar (Becton Dickinson and Company, Cockeysville, MD, USA) and incubated for $18-24 \mathrm{~h}$ at $37^{\circ} \mathrm{C}$. Standard microbiological technique were conducted to identify bacteria including colony morphology, Gram stain, and biochemical tests (Oxoid). Gram-positive cocci were identified based on their gram reaction, catalase and coagulase test results. Gram-negative rods were identified by performing a series of biochemical tests such as Kligler Iron Agar (KIA), Simon's citrate agar, Indole, urea, and motility. Blood collected for malaria and serology were analyzed within 30 minutes in their 
respective sections after collection. Samples were collected and processed by qualified laboratory technologist.

\section{Susceptibility Test}

Antimicrobial susceptibility testing was performed using disc diffusion on Müller-Hinton Agar according to Clinical Laboratory Standards Institute guidelines ${ }^{19}$. Bacterial isolates were tested against amoxicillinclavulanic acid $(30 \mu \mathrm{g})$, Amikacin $(30 \mu \mathrm{g})$, Ceftriaxone (30 $\mu \mathrm{g})$, Gentamicin (10 $\mu \mathrm{g})$, Imipenem (30 $\mu \mathrm{g})$, Trimethoprim-sulfamethoxazole $(23.75 \mu \mathrm{g} / 1.25 \mu \mathrm{g})$, Ciprofloxacin $(5 \mu \mathrm{g})$, Clindamycin $(2 \mu \mathrm{g})$, Erythromycin (15 $\mu \mathrm{g})$, Meropenem (10 $\mu \mathrm{g})$, Vancomycin $(30 \mu \mathrm{g})$, Tetracycline $(30 \mu \mathrm{g})$, Cefotaxime $(30 \mu \mathrm{g})$, Penicillin $(10 \mu \mathrm{g})$ and Chloramphenicol (30 $\mu \mathrm{g})$. All antibiotic discs were from Oxoid, ThermoFisher, and Scientific, USA. Antimicrobial sensitivity was reported as resistant, intermediate, and sensitive according to the Clinical Laboratory Standard Institute $^{19}$. The choice of antibiotic agents varied depending on the range of antibiotics available to the laboratory.

\section{Case definition}

BSI was defined as having either positive blood culture, malaria parasites, a positive serological result or any co-infections.

\section{Data analysis plan}

Data were analyzed using IBM SPSS Statistics for Windows, Version 22.0 (IBM Corp, Armonk, NY, USA). Cross-tabulation of categorical variables was calculated using Chi-square test $(\chi 2)$ while Fisher's exact test was used in cases when expected counts were $\leq 5$. The association between categorical predictors and the BSI was presented as odds ratio (OR) with 95\% confidence intervals (95\% CI) using logistic regression. Predictors significantly associated with BSI in the bivariate analysis were selected for multivariable analysis in the final model. A significance level $\leq 0.05$ was used throughout.

\section{Ethical Consideration}

Ethical approval to conduct this study was obtained from the Kilimanjaro Christian Medical University College Research and Ethics Review Committee (CRERC) with ethics certificate number 2472. Written consent was signed before filling the questionnaire. Permission to conduct the study was obtained from KCMC administration. Though all measures to protect the privacy and confidentiality was considered that neither name nor registration number was mentioned during data collection.

\section{RESULTS}

\section{Demographic characteristics of the study population}

A total of 200 patients were enrolled in the study, giving a response rate of $100 \%$. The modal age group was $0-5$ years with a frequency of $72(36 \%)$. More than half of the participants were female $110(55 \%)$. The majority of the participants lived in urban 119 $(59.5 \%)$. Most participants were self-employed 65 $(32.5 \%)$. Lastly, $58(29 \%)$ and $62(31 \%)$ had primary and secondary education respectively (Table 1 ).

\section{Prevalence and common etiologies of BSI infections}

Overall, $52 / 200(26 \%)$ of the participants were evident of having BSI infections. A total of 123 blood cultures were performed, 76/123 (61.8\%) from children (0-17 years) and $47 / 123(38.2 \%)$ from adults $>17$ years. Positive bacterial growth was observed in $41 / 123(33.3 \%)$ isolates with $18 / 123(14.6 \%)$ being significant for antimicrobial susceptibility testing. The number of pathogens recovered in the study period is presented in Figure 1. A total of 67 samples were subjected to serological tests. Out of these, $27(40.3 \%)$ were tested for Widal, 20(29.9\%) tested for Brucella and the remaining were tested for syphilis. The decisions for specific tests on samples were based on the clinical symptoms presented by the patient and the tests ordered by the attending clinician. A total of $10(37.0 \%)$ were positive for Widal while $2(10.5 \%)$ were positive for Brucella. Two of the participants had co-infection (typhoid and Brucellosis), Figure 2. A total of 51 participants were tested for malaria, only $1(2.0 \%)$ was found positive.

Antimicrobial susceptibility patterns of bacterial isolates Antibiotic-resistance patterns of bacteria against the antimicrobial agents is shown in Table 2. Coagulase Negative Staphylococcus (CoNS) showed highest resistance for Gentamicin 24 (96\%), Trimethoprim/sulfamethoxazole 24 (96\%), Imipenem $24(96 \%)$, Chloramphenicol $24(96 \%)$, Ciprofloxacin $23(92 \%)$, Clindamycin $23(92 \%)$ and Gentamycin 22 $(88 \%)$. Staphylococcus aureus showed the highest resistance for Meropenem 8 (88.8\%), Chloramphenicol $8(88.8 \%)$, Cefotaxime $6(66.6 \%)$, Tetracycline $6(66.6 \%)$, Amikacin 6 (66.6\%), Gentamicin $5(66.6 \%)$ and Imipenem $6(66.6 \%)$. The lowest level of resistance was observed in Ceftriaxone $1(11.1 \%)$.

\section{Risk factors associated with BSI}

The results of the bi- and multivariate analyses of the association between selected predictors and BSI are shown in Table 3. In the bivariate analysis: age of the participants, education level, infection risk, admission, length of admission and hospitalization showed an association with the outcome and were then selected to be included in the multivariate model. Only two factors remained independently associated with the occurrence of BSI after adjusting for confounding. Participants who reported having a diploma as their highest level of education had a higher odds of having BSIs compared to those with a degree $(\mathrm{AOR}=5.33$, 95\% CI=1.39-20.38; $\mathrm{P}=0.01)$. Participants with a stomachache had lower odds of having BSI compared to other patients with symptoms $(\mathrm{AOR}=0.22,5.33$, $95 \% \mathrm{CI}=0.05-0.97 ; \mathrm{P}=0.04)$. 
TABLE 1: Socio-demographic characteristics of the respondents ( $N=200$ )

\begin{tabular}{|c|c|c|c|}
\hline \multirow[b]{2}{*}{ Variable } & \multirow[b]{2}{*}{ n (\%) } & \multicolumn{2}{|c|}{ BSI infection } \\
\hline & & $\begin{array}{c}\text { Positive } \\
\text { n (\%) }\end{array}$ & $\begin{array}{c}\text { Negative } \\
n(\%)\end{array}$ \\
\hline \multicolumn{4}{|l|}{ Age group (years) } \\
\hline $0-5$ & $72(36.0)$ & $26(49.1)$ & $46(31.3)$ \\
\hline $6-17$ & $7(3.5)$ & $1(1.9)$ & $6(4.1)$ \\
\hline $18-45$ & $69(34.5)$ & $17(32.1)$ & $52(35.4)$ \\
\hline More than 45 & $52(26.0)$ & $9(17.0)$ & $43(29.3)$ \\
\hline \multicolumn{4}{|l|}{ Locality } \\
\hline Urban & $119(59.5)$ & $31(58.5)$ & $88(59.9)$ \\
\hline Rural & $81(40.5)$ & $22(41.5)$ & $59(40.1)$ \\
\hline \multicolumn{4}{|l|}{ Sex } \\
\hline Male & $90(45.0)$ & $26(49.1)$ & $64(43.5)$ \\
\hline Female & $110(55.0)$ & $27(50.9)$ & $83(56.5)$ \\
\hline \multicolumn{4}{|l|}{ Education level } \\
\hline Illiterate & $4(2.0)$ & $0(0.0)$ & $4(2.7)$ \\
\hline Primary level & $58(29.0)$ & $19(35.8)$ & $39(29.5)$ \\
\hline Secondary level & $62(31.0)$ & $17(32.1)$ & $45(30.6)$ \\
\hline Diploma level & $32(16.0)$ & $2(3.8)$ & $30(20.4)$ \\
\hline Degree level & $32(16.0)$ & $11(20.8)$ & $21(14.3)$ \\
\hline Others & $12(6.0)$ & $4(7.5)$ & $8(5.4)$ \\
\hline \multicolumn{4}{|l|}{ Occupation status } \\
\hline Employed & $61(30.5)$ & $13(24.5)$ & $48(32.7)$ \\
\hline Unemployed & $43(21.5)$ & $12(22.6)$ & $31(21.1)$ \\
\hline Student & $31(15.5)$ & $7(13.2)$ & $24(16.3)$ \\
\hline Self-employed & $65(32.5)$ & $21(39.6)$ & $44(29.9)$ \\
\hline \multicolumn{4}{|l|}{ Income } \\
\hline Less than $300,000 /=$ & $62(31.0)$ & $18(34.0)$ & 44 (29.9) \\
\hline $300,000 /=$ to $1,000,000 /=$ & $57(28.5)$ & $14(26.4)$ & $43(29.3)$ \\
\hline More than $1,000,000 /=$ & $4(2.0)$ & $1(1.9)$ & $3(2.0)$ \\
\hline No income & $77(38.5)$ & $20(37.7)$ & $57(38.8)$ \\
\hline
\end{tabular}

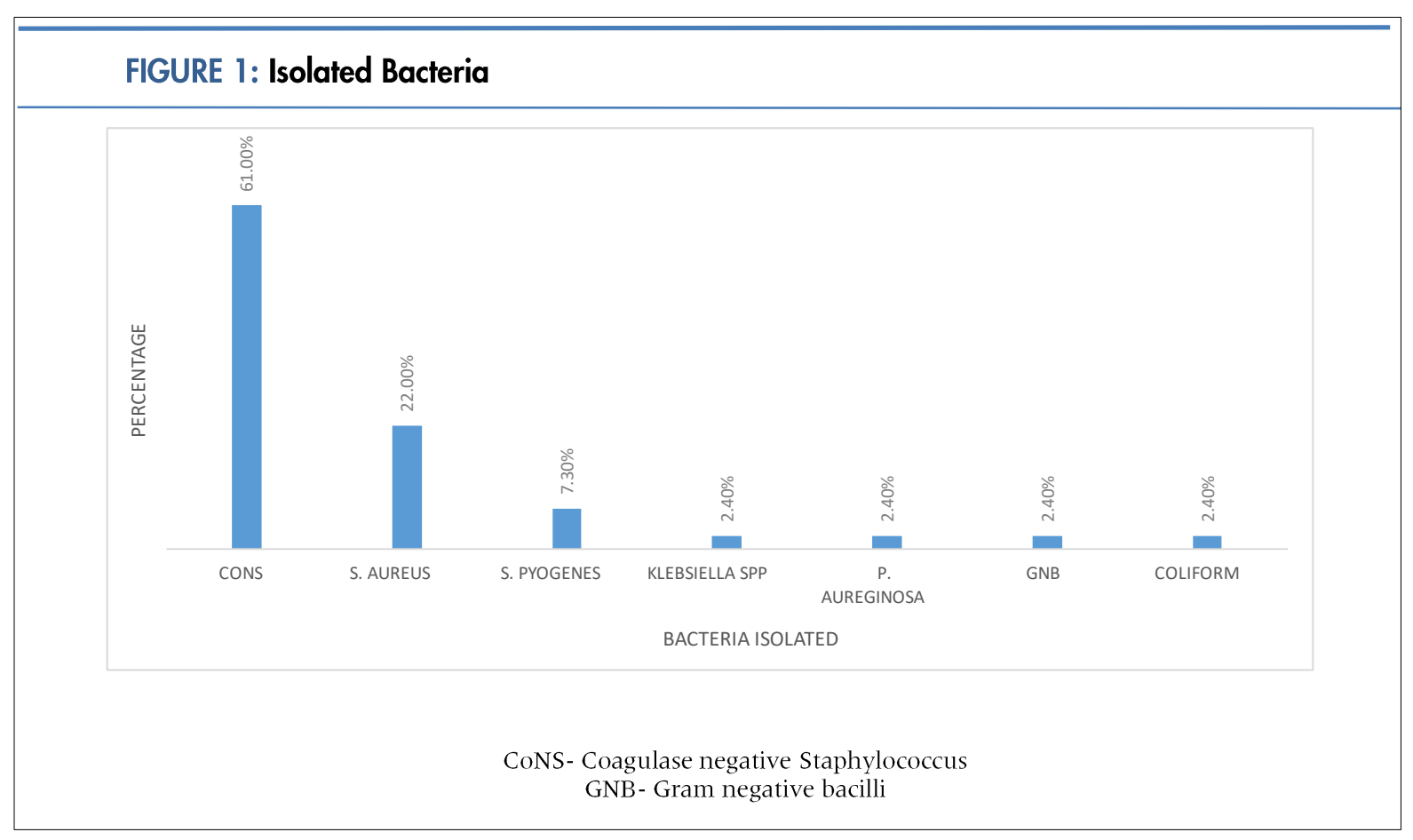




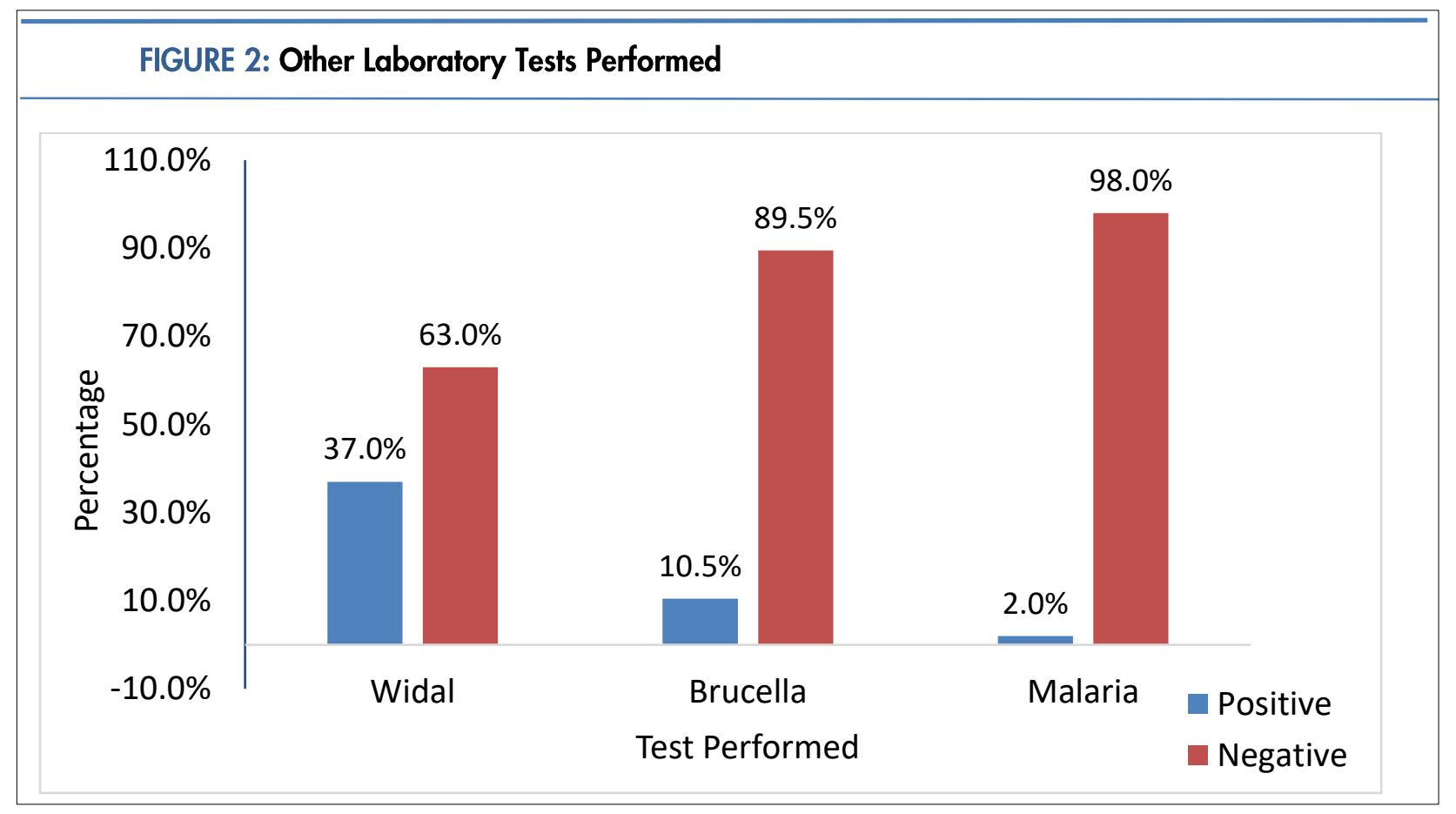

TABLE 3: Factors associated with BSI

\begin{tabular}{|c|c|c|c|c|}
\hline Variable & COR & P-value & AOR & P-valu \\
\hline \multicolumn{5}{|l|}{ Age group (years) } \\
\hline $0-5$ & $0.37(0.15-0.87)$ & 0.02 & $1.29(0.17-9.41)$ & 0.7 \\
\hline $6-17$ & $1.25(0.13-11.74)$ & 0.8 & $3.77(0.25-55.05)$ & 0.3 \\
\hline $18-45$ & $0.69(0.27-1.72)$ & 0.4 & $0.61(0.20-1.89)$ & 0.3 \\
\hline More than 45 & Reference & & Reference & \\
\hline \multicolumn{5}{|l|}{ Locality } \\
\hline Urban & $1.10(0.58-2.10)$ & 0.7 & & \\
\hline Rural & Reference & & & \\
\hline \multicolumn{5}{|l|}{ Sex } \\
\hline Male & $0.84(0.44-1.59)$ & 0.6 & & \\
\hline Female & Reference & & & \\
\hline \multicolumn{5}{|l|}{ Education level } \\
\hline Primary level & $1.12(0.45-2.73)$ & 0.8 & $2.37(0.69-8.07)$ & 0.1 \\
\hline Secondary level & $1.47(0.60-3.61)$ & 0.3 & $2.75(0.89-8.53)$ & 0.07 \\
\hline Diploma level & $5.04(1.44-17.58)$ & 0.01 & $5.33(1.39-20.38)$ & 0.01 \\
\hline Degree level and above & Reference & & Reference & \\
\hline \multicolumn{5}{|l|}{ Occupation } \\
\hline Employed & $1.93(0.86-4.32)$ & 0.1 & $1.69(0.59-4.79)$ & 0.3 \\
\hline Unemployed & $1.65(0.78-3.47)$ & 0.1 & $1.44(0.59-3.49)$ & 0.4 \\
\hline Self-employed & Reference & & & \\
\hline
\end{tabular}

Income

Less than $300,000 /=$ $300,000 /=$ to $1,000,000 /=$

More than $1,000,000 /=$
$0.91(0.09-9.11)$

$1.02(0.09-10.65)$

Reference
0.9

0.9

\section{Symptoms}




\begin{tabular}{|c|c|c|c|c|}
\hline Fever & $0.78(0.35-1.75)$ & 0.5 & $0.67(0.27-1.69)$ & 0.4 \\
\hline Headache & $1.56(0.44-5.52)$ & 0.4 & $1.14(0.27-4.84)$ & 0.8 \\
\hline Stomachache & $0.44(0.13-1.53)$ & 0.1 & $0.22(0.05-0.97)$ & 0.04 \\
\hline Diarrhea & $0.44(0.06-3.01)$ & 0.4 & $0.22(0.02-2.13)$ & 0.1 \\
\hline Others* & Reference & & & \\
\hline \multicolumn{5}{|l|}{ Co-morbidities } \\
\hline Diabetes & $1.78(0.20-16.67)$ & 0.6 & & \\
\hline Hypertension & $0.89(0.26-2.98)$ & 0.8 & & \\
\hline Cancer & $0.35(0.49-2.60)$ & 0.3 & & \\
\hline Others & $1.06(0.27-4.13)$ & 0.9 & & \\
\hline None & Reference & & & \\
\hline \multicolumn{5}{|l|}{ Infection risks $(\mathrm{N}=87)$} \\
\hline Urinary catheter & $0.59(0.14-2.45)$ & 0.4 & $0.80(0.15-4.13)$ & 0.7 \\
\hline Intravascular catheter & $0.43(0.22-0.84)$ & 0.01 & $0.74(0.29-1.85)$ & 0.5 \\
\hline None & Reference & & Reference & \\
\hline \multicolumn{5}{|l|}{ Admission } \\
\hline Yes & $0.32(0.16-0.65)$ & 0.02 & $0.12(0.005-3.10)$ & 0.2 \\
\hline No & Reference & & Reference & \\
\hline \multicolumn{5}{|l|}{ Duration of hospitalization } \\
\hline Less than 7 days & $0.41(0.19-0.87)$ & 0.02 & $1.35(0.02-68.09)$ & 0.8 \\
\hline 7 to 14 days & $0.29(0.11-0.73)$ & 0.009 & $0.71(0.01-39.24)$ & 0.8 \\
\hline More than 14 days & $0.18(0.04-0.82)$ & 0.02 & $0.46(0.006-34.71)$ & 0.7 \\
\hline Not admitted & Reference & & Reference & \\
\hline \multicolumn{5}{|l|}{ Ward } \\
\hline Medical & $0.75(0.26-2.15)$ & 0.5 & $4.70(0.35-62.17)$ & 0.2 \\
\hline Surgical & $0.34(0.07-1.57)$ & 0.1 & $3.22(0.17-59.01)$ & 0.4 \\
\hline Pediatric & $0.32(0.15-0.67)$ & 0.02 & $1.31(0.07-22.70)$ & 0.8 \\
\hline Others & Reference & & Reference & \\
\hline
\end{tabular}

COR: Crude Odds Ratio, AOR: Adjusted Odds Ratio

* Other symptom such as Rashes and vomit/nausea

\section{DISCUSSION}

This study was conducted among patients who visited KCMC. The study aimed at determining the prevalence of BSIs, associated factors, and antibiotic susceptibility. The prevalence of positive blood culture in this study was found to be $14.6 \%$ which is comparable to other studies conducted in the same region (Zanzibar $(14.0 \%)^{20}$, Muhimbili-Tanzania $(13.4 \%){ }^{6}$ A higher prevalence was observed in a study done in Malawi which reported a prevalence of $30 \%$, also difference may be due to different study population whereby it was specific to adults only who presented with fever ${ }^{22}$. Coagulase Negative Staphylococcus $(61.0 \%)$ was the most predominant bacteria isolated among participants with bacterial infections, followed by Staphylococcus aureus $(22.0 \%)$. This pattern is similar to a study conducted in Dar Es Salaam Tanzania which also reported that Staphylococcus aureus as the most common cause of BSIs ${ }^{6}$. CoNS was also reported to be the most isolated bacteria in a study conducted in Ghana ${ }^{23}$, while Staphylococcus aureus was the second most isolated bacteria among patients with BSI in Ghana $^{23}$ and Malawi ${ }^{24}$. Salmonella typhi was the most common organism isolated among the positive participants. Different studies have also reported
Salmonella being a major cause of infection in Bangladesh $(36.9 \%)^{21}$, Nepal $(71 \%)^{25}$ and Malawi ${ }^{24}$.

Coagulase Negative Staphylococcus showed the highest rates of drug resistance to Trimethoprim/sulfamethoxazole, Imipenem, Chloramphenicol, Ciprofloxacin, Clindamycin and Gentamycin. Similarly, a study in India reported that Ciprofloxacin and Clindamycin was resistance to CoNS $^{26}$. Staphylococcus aureus showed the highest rates of resistance for gentamicin $(66.6 \%)$. This finding is comparable to similar reports from Brazill ${ }^{27}$, India ${ }^{26}$ and Senegal ${ }^{28}$. In contrary, a study conducted in Zanzibar reported high susceptibility of Staphylococcus aureus to Cefotaxime and Tetracycline ${ }^{20}$. In general, our findings suggest that the that isolates found on the Tanzanian mainland are quite similar to those found elsewhere ${ }^{26-28}$. However, we noted small differences with a study conducted in Zanzibar ${ }^{20}$. It is possible that the smaller population and more limited circulation of people reduces the transmission of some strains of the pathogen. Based on the observed results in this study, treatment should follow microbiology investigations and not be solely based on clinical symptoms. This will improve the accuracy of diagnosis and support the reduction in the occurrence of AMR. Further efforts 
are needed to raise awareness amongst the community and healthcare workers as to the rapid increase in drug resistance and advocate on the antimicrobial stewardship program in these settings.

The education of individuals can help tackling the spread of disease, given that educated people may be more aware how diseases circulate in their community. The probability of having or dying from infections such as malaria is inversely related to income and education $^{29}$. However, this was not the case in this study, our results suggest that those with a higher level of education (diploma) were more likely to have a BSI. It is possible that this result is caused by a selection bias, the study was hospital based, so it is possible that those who are more educated may have better health seeking behavior and therefore more likely to attend hospital and then be included in this study. A study conducted in America showed that education level was a significant factor associated with BSIs for more than $30 \%$ of participants ${ }^{18}$. Though lacking statistical significance our findings suggest that participants with diabetes were $78 \%$ more likely to have BSIs compared to others. Our sample size was small, and this may have reduced our power to detect an association. Further studies should be conducted to investigate this association. Finally, our study suggests that increased efforts should be taken to reduce opportunities for infection.

The findings of this study should be considered with the following limitations in mind. Firstly, a single blood culture specimen was collected from each patient, therefore it was not possible to determine if the patients with CoNS isolation had true bacteremia or the finding was due to skin contamination. The data for this study were obtained for three months between April and June 2019, and therefore seasonal variations in the frequency of BSI and causative microorganisms could not be assessed.

\section{CONCLUSION}

We identified a high prevalence of BSIs across all age groups and more commonly associated with education level. Staphylococcus spp was the most commonly isolated organism among patients with BSIs and exhibited high levels of AMR. We recommend that laboratory investigations and susceptibility tests of isolated bacteria should guide treatment protocols, and these should not be solely based on clinical symptoms. Acknowledgment

The authors would like to thank respondents of this study who voluntarily took part in this study.

Acknowledgement: The authors would like to thank respondents of this study who voluntarily took part in this study

\section{Financial support}

Nil
Conflict of interest

There was no conflict of interest

\section{REFERENCES}

1. Crump A., Morrissey A., Nicholson W., et al. Etiology of severe non-malaria febrile illness in Northern Tanzania: a prospective cohort study. PLoS Negl Trop Dis. 2013;7(7):1-9.

2. Reddy EA, Shaw A V, Crump JA. Community-acquired bloodstream infections in Africa: a systematic review and metaanalysis Elizabeth. Lancet Infect Dis. 2010;10(6):70072-70074. doi:10.1016/S1473-3099(10)70072-4.

3. Nichols C, Cruz Espinoza LM, Von Kalckreuth V, et al. Bloodstream infections and frequency of pretreatment associated with age and hospitalization status in Sub-Saharan Africa. Clin Infect Dis. 2015;61(Suppl 4):S372-S379. doi:10.1093/cid/civ730

4. Blomberg B, Manji KP, Urassa WK, et al. Antimicrobial resistance predicts death in Tanzanian children with bloodstream infections: A prospective cohort study. BMC Infect Dis. 2007;7:1-14. doi:10.1186/1471-2334-7-46

5. Manyahi J, Kibwana U, Mgimba E, Id MM. Multi-drug resistant bacteria predict mortality in bloodstream infection in a tertiary setting in Tanzania. PLoS One. 2020;15(3):1-11. doi:10.1371/journal.pone.0220424

6. Moyo S, Aboud S, Kasubi M, Maselle SY. Bacteria isolated from bloodstream infections at a tertiary hospital in Dar es Salaam, Tanzania - antimicrobial resistance of isolates. South African Med J. 2010;100(12):835-838. doi:10.7196/SAMJ.4186

7. Seni J, Mwakyoma AA, Mashuda F, et al. Deciphering risk factors for blood stream infections, bacteria species and antimicrobial resistance profiles among children under five years of age in North- Western Tanzania : a multicentre study in a cascade of referral health care system. BMC Pediatr. 2019;19(32):1-11.

8. Kajeguka DC, Nambunga PP, Kabissi F, et al. Antimicrobial resistance patterns of phenotype Extended Spectrum BetaLactamase producing bacterial isolates in a referral hospital in northern Tanzania. Tanzan J Health Res. 2015;17(3):1-8. doi: $10.4314 /$ thrb.v17i3.\%c

9. Moremi N, Claus H, Mshana SE. Antimicrobial resistance pattern: A report of microbiological cultures at a tertiary hospital in Tanzania. BMC Infect Dis. 2016;16(1). doi:10.1186/s12879016-2082-1

10. Kumburu HH, Sonda T, Mmbaga BT, et al. Patterns of infections, aetiological agents and antimicrobial resistance at a tertiary care hospital in northern Tanzania. Trop Med Int Heal. 2017:22(4):454-464. doi:10.1111/tmi.12836

11. Seni J, Sweya E, Mabewa A, Mshana SE, Gilyoma JM. Comparison of antimicrobial resistance patterns of ESBL and non ESBL bacterial isolates among patients with secondary peritonitis at Bugando Medical Centre, Mwanza - Tanzania. BMC Emerg Med. 2016;16(1). doi:10.1186/s12873-016-0106-1

12. Marando R, Seni J, Mirambo MM, et al. Predictors of the extended-spectrum-beta lactamases producing Enterobacteriaceae neonatal sepsis at a tertiary hospital, Tanzania. Int J Med Microbiol. 2018;308(7):803-811. doi: 10.1016/j.ijmm.2018.06.012

13. Kumburu HH, Sonda T, Leekitcharoenphon P, et al. Hospital Epidemiology of Methicillin-Resistant Staphylococcus aureus in a Tertiary Care Hospital in Moshi, Tanzania, as Determined by Whole Genome Sequencing. Biomed Res Int. 2018;2018:1-12. doi:10.1155/2018/2087693

14. Blomberg B. Antimicrobial resistance in bacterial infections in urban and rural Tanzania. BMC Public Health. 2007. doi:10.1186/1471-2334-4-35

15. Kajeguka DC, Kaaya RD, Mwakalinga S, et al. Prevalence of dengue and chikungunya virus infections in north-eastern Tanzania: a cross sectional study among participants presenting with malaria-like symptoms. BMC Infect Dis. 2016;16(183):1-9. doi:DOI 10.1186/s12879-016-1511-5

16. Crump JA, Gove S, Parry CM. Management of adolescents and adults with febrile illness in resource limited areas. BMJ. 2011;343(d4847). doi:10.1136/bmj.d4847

17. Kajeguka DC, Desrochers RE, Mwangi R, et al. Knowledge and practice regarding dengue and chikungunya: a cross-sectional study among Healthcare workers and community in Northern Tanzania. Trop Med Int Heal. 2017;22(5):583-593. 
doi:10.1111/tmi. 12863

18. Rybak MJ, Zasowski EJ, Jorgensen SCJ. Risk Factors for Bloodstream Infections Among an Urban Population with Skin and Soft Tissue Infections: A Retrospective Unmatched CaseControl Study. Infect Dis Ther. 2018:1-11. doi:10.1007/s40121018-0227-9

19. CLSI. Clinical and Laboratory Standards Institute. Performance Standards for Antimicrobial Susceptibility Testing: CLSI AST News Update.2018:1-3.

20. Onken A, Said AK, Melissa J, Jenum PA. Prevalence and Antimicrobial Resistance of Microbes Causing Bloodstream Infections in Unguja, Zanzibar. PLoS One. 2015;10(12):1-10. doi:10.1371/journal.pone.0145632

21. Abmed D, Nahid A, Sami AB, et al. Bacterial etiology of bloodstream infections and antimicrobial resistance in Dhaka. Antimicrob Resist Infect Control. 2017;6(2):1-11. doi:10.1186/s13756-016-0162-z

22. Archibald LK, Mcdonald LC, Nwanyanwu O, et al. A HospitalBased Prevalence Survey of Bloodstream Infections in Febrile Patients in Malawi: Implications for Diagnosis and Therapy. J Infect Dis. 2000;181:1414-1420.

23. Deku JG, Dakorah MP, Lokpo SY, et al. The Epidemiology of Bloodstream Infections and Antimicrobial Susceptibility Patterns: A Nine-Year Retrospective Study at St. Dominic Hospital, Akwatia, Ghana. J Trop Med. 2019;2019:1-10. doi:10.1155/2019/6750864

24. Musicha P, Cornick JE, Bar-Zeev N, et al. Trends in antimicrobial resistance in bloodstream infection isolates at a large urban hospital in Malawi (1998-2016): a surveillance study. Lancet Infect Dis. 2017:17(10):1042-1052. doi:10.1016/S1473-3099(17)30394-8

25. Bhandari P, Manandhar S, Shrestha B, Dulal N. Etiology of bloodstream infection and antibiotic. Asian $\mathrm{J}$ Med Sci. 2016:7(2):71-75. doi:10.3126/ajms.v7i2.13444

26. Singh A, Venkatesh V, Singh R, Singh M. Bacterial and antimicrobial resistance profile of bloodstream infections: A hospital-based study. CHRISMED J Heal Res. 2014;1(3):140144. doi:10.4103/2348-3334.138881

27. Monteiro A de S, Pinto BLS, Monteiro J de M, et al. Phylogenetic and molecular profile of Staphylococcus aureus isolated from bloodstream infections in northeast Brazil. Microorganisms. 2019;7(210):1-14. doi:10.3390/microorganisms 7070210

28. Lakhe NA, Sylla K, Mbaye KD, et al. Bacteremia: Profile and Antibiotic Resistance at the Infectious and Tropical Diseases Clinic in Fann Hospital, Dakar, Senegal. J Infect Dis Ther. 2018;6(1):1-8. doi:10.4172/2332-0877.1000348

29. Tusting LS, Willey B, Lucas $\mathrm{H}$, et al. Socioeconomic development as an intervention against malaria: A systematic review and meta-analysis. Lancet. 2013;382:963-972. doi:10.1016/S0140-6736(13)60851-X

30. Dinan TG, Cryan JF. Microbes Immunity and Behavior: Psychoneuroimmunology Meets the Microbiome. Neuropsychopharmacology. 2017;42(1):178-192. doi:10.1038/npp.2016.103

Peer Reviewed

Competing Interests: None declared.

Received: 8/28/2020; Accepted: 2/22/2021

Cite this article as: Mauki II, William JD, Mlay HL, Saro TA, Saringe SO, Mkumbaye SI, Kajeguka DC. Etiologies of bloodstream infection and antimicrobial resistance: A cross sectional study among patients in a tertiary hospital, Northern Tanzania. E Afr Sci. 2021;3(1):104-111. http://doi.org/10.24248/EASCi-D-20-00012

(-) Mauki II et al. This is an open-access a t ticle distributed under the terms of the Creative Commons Attribution License, which permits unrestricted use, distribution, and reproduction in any medium, provided the original author and source are properly cited. To view a copy of the license, visit http://creativecommons.org/licens- es/by/4.0/. When linking to this article, please use the following permanent link: http://doi.org/10.24248/EASci-D-20-00012 